\title{
Questions and Answers in EBD Volumes 3 and 4
}

\author{
Derek Richards \\ Editor, Evidence-Based Dentistry \\ Evidence-Based Dentistry (2003) 4, 94-97. doi:10.1038/sj.ebd.6400226
}

Previously ${ }^{1}$ we have outlined the Questions and Answers addressed by summaries in EBD volumes one and two. Here we list a further 84 questions, answers and evidence levels of areas addressed in EBD volumes 3 and 4 . Evidence levels ${ }^{2}$ are only given for those papers achieving level 3A and above.

Question

\section{Cariology}

Is a glass ionomer fissure sealant effective in reducing occlusal caries?

Are there clear agreed definitions for early or severe childhood caries?

\section{Cosmetic Dentistry}

Are dentist prescribed home applied tooth whitening agents effective?

\section{Dental Education}

Are computer-aided learning (CAL) programmes effective in teaching dental students?

\section{Dental Implants}

Do hydroxyapatite implants survive as long as titanium ones?

Does the use of dental implants in edentulous patients result in better quality of life compared with conventional treatment?

\section{Endodontics}

In patients with radiographically determined failure of surgical endodontics what is the success rate of repeat surgery?

\section{Infection Control}

How well do dental team members follow infection control guidelines?

\section{Oral Cancer}

What is the discriminatory ability of visual oral cancer screening?

Does information about oral cancer encourage primary care patients to accept oral cancer screening?

What are the risk factors associated with the development of oral cancer in young people?

Is human papilloma virus (HPV) a risk factor for oral cancer?

\section{Oral Medicine}

Which antifungal agent is the most effective in oropharyngeal candidiasis?

Does systemic penicillin improve pain control in patients with irreversible pulpitis?

What are the features of cluster headaches?
Answer

\section{Evidence}

level

Glass ionomer sealant has a caries-preventive effect

1B

Volume

and page number

No - There is a clear need for Internationally accepted definitions

On average a 2 shade guide improvement in colour can

The review suggests that survival rates for both sets of implants are similar, but that more work and better quality reporting is required

Implant patients had better quality of life in this short term follow-up study

Discriminatory ability is high irrespective of grade and training

A simple leaflet in primary health care setting can change knowledge about oral cancer and increase intention to go for screening and reduce anxiety about screening

Oral cancer has increased in people aged under 45 . Tobacco and alcohol use may be involved but clear risk factors are yet to be identified. More work is required to identify risk factors in younger people

Both oral dysplasia and oral cancer are more commonly associated with HPV than normal mucosa

A number of effective agents are available. Because of drug resistance it seems sensible to limit their use to treatment of clinically evident oral candidal infection Evidence suggests that use of antibiotics does not help in pain relief of irreversible pulpitis

The review provides encouraging results and guidelines 
In patients with hypertension does use of epinephrine containing anaesthetic solution or epinephrine impregnated retraction cord produce adverse cardiovascular outcomes?

Are people with HIV/AIDS at greater risk of complications from intra-oral dental procedures than HIVnegative patients?

\section{Oral Surgery}

Is ropivacaine as effective as bupivacaine?

Is it necessary to stop low-dose aspirin prior to oral surgery?

Do you need to discontinue anticoagulants before routine dental extractions?

Does the use of a lingual retractor increase the risk of nerve damage during third molar extraction?

What treatment is best for people with odontogenic keratocysts?

\section{Orthodontics}

What is the most effective adhesive for bonding orthodontic brackets to teeth?

What is the optimal force required for orthodontic tooth movement?

Does orthodontic treatment have any consequences for the long-term development of signs and symptoms of temporomandibular disorders?

Is a hydrophilic or conventional primer more effective in orthodontic bonding?

Are there characteristic features of the craniofacial skeleton in patients of children who have orofacial clefting?

\section{Paediatric Dentistry}

In children with pulpally involved primary molars what pulp treatment techniques are effective in retaining the tooth and avoiding long-term sequelae?

In children with high-risk of dental caries is supervised toothbrushing effective in reducing disease?

Are preformed metal crowns (PMCs) more effective than amalgam restorations in primary molars?

\section{Periodontology}

Is guided tissue regeneration effective in treatment of infra-bony pockets?

Is potassium nitrite containing toothpaste effective in reducing dentine hypersensitivity?

In patients with intrabony defects is surgery with enamel matrix derivative (EMD) more effective than other treatments?

Is the use of grafting biomaterials or biological agents and open flap debridement (OFD) more effective than OFD alone for deep intra-osseous pockets?

Do adjunctive systemic antimicrobials improve clinical outcome of scaling and root planing in treatment of periodontitis?

In people with gingivitis and periodontitis are powered toothbrushes more effective than manual ones in reducing bleeding and inflammation?

When treating chronic periodontitis, are machinedriven more effective than hand instruments?

What is the quality of randomised controlled trials (RCT) in periodontology?

In patients who have periodontitis, what is the effect of for appropriate management

Epinephrine in dental local anaesthetics has little effect on blood pressure and heart rate in hypertensive patients at doses equivalent to one or two cartridges. There is no reliable evidence concerning the safety of epinephrine in gingival retraction cord in hypertensive patients

The available evidence suggests that complication from intra-oral procedure for HIV/AIDS patients is low so they may be treated in dental practice as other patients

This small trial showed similar effectiveness during infiltration anaesthesia - more studies are needed in regional block anaesthesia

This trial suggests that it is not necessary to stop lowdose aspirin prior to minor oral surgery

The study in a hospital situation showed that there was no need to stop anticoagulation in the International normalised ratio was $<4.1$

Retractor use was associated with an increase in temporary nerve damage, but neither protective nor detrimental with respect to permanent damage Current evidence suggests that enucleation with application of Carnoy's solution provides best outcome but more research needed

There is no clear evidence to provide an answer at present

There is currently no evidence identifying the magnitude of an optimum orthodontic force

This paper adds weight to the evidence that orthodontic treatment does not predispose patients to TMD later in life and that most malocclusions, with the exception of lateral displacements on closing and/or unilateral crossbite, are not associated with the development of TMD.

This trial showed increased bracket failure with hydrophilic primer but involved small numbers of patients The quality of the available data is limited but suggests that there are characteristic features

No reliable evidence to support the superiority of one particular treatment method.

Targeted supervised toothbrushing with home support can produce significant reduction in caries levels. Evidence suggests that PMCs are more effective than amalgam restoration but more research required

Some evidence for increasing attachment loss but factors for success and failure not clear

No strong evidence to support effectiveness

Currently the evidence for possible effect of EMD in treatment of intrabony defects is small

Currently there is no evidence to support the use of these graft materials and biological agents for treating periodontal intra-osseous pockets

Periodontal treatment may be more effective with adjunctive antimicrobials

Powered brushes particularly counter-rotational and oscillation rotating brushes are more effective

There are no obvious clinical differences but ultrasonic/ sonic instruments provide a small time saving. More research is needed in particular regarding patient related benefits.

The quality of RCT reporting in periodontology (and probably other dental disciplines) needs to improve This study shows a negative effect of smoking on 
smoking on the response to access flap surgery?

In patients with moderate to advanced periodontal disease, is scaling plus minocycline microspheres more effective than scaling alone?

In patients having intra-bony periodontal defects treated with enamel matrix proteins, does the use of post surgical antibiotics improve outcome?

In patients undergoing periodontal scaling and root planning, is an anaesthetic gel effective in producing pain relief?

Is subantimicrobial dose doxycycline in conjuction with subgingival scaling and root planning effective in treatment of adult periodontitis?

In patients with buccal gingival recession is periodontal plastic surgery effective?

What is the prevalence of root sensitivity after periodontal therapy?

Is subgingival debridement clinically effective in people who have chronic periodontitis?

Is periodontitis associated with coronary heart disease, preterm birth and low birth weight?

Is guided tissue regeneration (GTR) more effective than connective tissue grafting (CTG) in the treatment of gingival recession defects?

Is it possible to distinguish between chronic and aggressive periodontitis based on presence of particular periodontal pathogens?

In patients with periodontal disease are residual probing depth, bleeding on probing and furcation status predictors of further attachment loss?

Which anti-infective therapies are effective in periimplantitis?

\section{Preventive Dentistry}

Are fluoride mouth rinses effective in preventing caries?

Should dentists provide tobacco cessation advice?

In children and adolescents, is brushing with fluoride toothpaste more effective than a non fluoride paste? Are powered toothbrushes more effective than manual brushes at reducing levels of plaque and gingivitis in people who have normal manual dexterity?

Is a prebrushing mouth rinse an effective adjunct to oral hygiene?

What is the most effective intervention for maintaining health tissue around dental implants?

Are fluoride gels applications effective at reducing caries in children?

In children under 16 is fluoride varnish effective in preventing caries?

In people with primary root caries, is 5000 ppm fluoride dentifrice more effective than $1100 \mathrm{ppm}$ dentifrice in the reversal of lesions

Does chewing xylitol gum result in suppression of mutans streptococci?

In patients with orthodontic appliances are powered toothbrushes more effective than manual brushes? In susceptible teenagers is chlorhexidine varnish more effective than fluoride varnish in preventing caries? Is chlorhexidine varnish effective in reducing caries in adults with reduced salivary flow?

Does water fluoridation reduce incidence of caries? gingival surgery but more studies are required to clarify the real effect

This study shows a statistical benefit but further studies would be needed to show a convincing clinical benefit

No advantage in using systematic antibiotics in this type

No clear answer yet

The trial did not provide a definitive answer

With the exception of free gingival grafts and lateral sliding flaps, most surgical techniques are successful at producing root coverage for Miller's Class I and II defects

Current best evidence suggest over half of all patients suffer with root sensitivity after periodontal treatment subgingival debridement and plaque control is effective in reducing pocket depth and improving attachment level

While studies do show an association there is no evidence to recommend periodontal treatment to reduce coronary heart disease or preterm low birth weight

There is no clinical advantage in using GTR over CTG in treatment of gingival recession defects

At present there is no evidence to support differentiation based on particular pathogenic bacteria

Residual probing depths may be an indication of further disease progression

Evidence is absent for anti-infective treatment for periimplantitis so good quality trials are needed

Fluoride rinses reduce caries.

Fluoride rinses are more effective in high caries areas. Background fluoride use has little influence on the preventive effect of fluoride rinses.

Most practitioners believed that they should offer tobacco cessation advice but thought that patients did not expect it

Fluoride toothpaste prevents caries and has a greater effect in people with more disease

Powered brushes with rotation-oscillation action achieve a modest benefit over manual brushes

The clinical benefit is limited

Little reliable evidence about what is the most reliable evidence. No evidence that powered brushes better than manual but weak evidence to support adjunctive use of Listerine Mouth wash

Application of fluoride gels professionally or self-applied are associated with a substantial reduction in caries increment

Fluoride varnish produces a substantial caries inhibiting effect in the deciduous and permanent teeth of children The higher concentration had a greater effect but this is a single study of only 6 months duration

Chewing xylitol gum by mothers with high mutans streptococci produces long-term suppression in their children. Any clear clinical impact of this is yet to be demonstrated

Some types of powered brushes show a modest benefit

This study does not provide evidence that chlorhexidine varnish offers any advantage over fluoride varnish Chlorhexidine varnish has a positive effect but further studies needed

Fluoridation reduces caries but quality of evidence not
$1 \mathrm{~A}$

$1 \mathrm{~A}$

$1 \mathrm{~A}$

$1 \mathrm{~A}$

$3.64-65$

$1 \mathrm{~A}$

3.105-106

$1 B$

$1 \mathrm{~B}$

1B

4.27

$1 \mathrm{~B}$

4.12

$1 \mathrm{~B}$

3.53

$3 \mathrm{~A}$ 
Is fluoridation associated with other negative effects?

Is fluoridation associated with cancer?

Are there differences between natural and artificial fluoridation?

Is fluoridation associated with bone fractures?

Is fluoridation associated with fluorosis?

Does fluoridation result in a reduction of caries across social groups bringing equity

Does water fluoridation have an effect over and above that offered by alternative interventions and strategies?

\section{Radiography}

Can the use of a computer-assisted learning programme improve the ability of dental students to detect caries from bitewing radiographs?

\section{Restorative Dentistry}

When patients require posterior restorations are ceramic restorations more effective than other restorative materials?

Are dental appliances effective in the treatment of obstructive sleep apnoea?

Which material provided the best clinical properties for temporary restorations?

Which filing material last longest?

\section{Temporomandibular Disorders}

Is occlusal adjustment effective for the treatment and prevention of temporomandibular joint disorders?

Is arthrocentesis with sodium hyaluronate more effective than arthrocentesis alone in people with temporomandibular derangements

Is minimal treatment effective in people who have anterior disk displacement?

What are the long term predictors of signs and symptoms of temporomandibular disorders? high so it is difficult to give a good estimate of effect

The available evidence is insufficient to reach conclusions

The available evidence showed no association but the evidence was of low quality

The available evidence is insufficient to reach conclusions

The available evidence showed no association but the evidence was of low quality

There was an association between fluorosis and fluoridation and a dose-response was identified but the quality of the studies was low

Some evidence of a beneficial effect but quality of evidence and number of studies small

Evidence not of high quality but suggests an effect over and above other sources of fluoride (eg toothpaste)

Little evidence to support any difference in clinical performance of ceramic restorations over other posterior restorations

Dental appliances may be a useful adjunct in the treatment of obstructive sleep apnoea

No difference were noted between material in this small study

Amalgam had the best clinical performance but the quality of the included studies in the review was mediocre and there was little direct comparison of materials

At present there is no evidence to support occlusal treatment in treatment or prevention of temporomandibular joint disorders

Patients in both groups benefited from treatment with suggestion that those having sodium hyaluronate did better but trial is with small number of patients.

Results suggest that patients will normally improve with minimal treatment

Parafunction, tooth wear, TMJ clicking and deep overbite are possible predictors, but their predictive value require further study

1. Lawrence A, Richards D. Questions answered in EBD Volumes 1 and 2. EBD 2002; 3:27-29.

2. Richards D. Not all evidence is created equal — so what is good evidence. EBD 2003; 4:17-18. 